\section{IGHV1 status in chronic lymphocytic leukemia identify ethnic groups with an aggressive clinical course (Comment to Giudice ID, Foà $R$. Haematologica. 2019;104(2):219-221)}

We have read with great interest the editorial by Del Giudice et al. concerning the history of immunoglobulin heavy chain variable region (IGHV1) mutations based on a prognostic approach in chronic lymphocytic leukemia (CLL) patients. ${ }^{1}$ In their editorial the authors reviewed the importance of IGHV1 mutations in CLL in the light of the results obtained by the ERIC group, published in the same issue of Haematologica. ${ }^{2}$ They underlined that the IGHV1 mutational status, additional to TP53 deletions/mutations, is still the most robust prognostic biomarker. In fact, the patients harboring a high number of somatic mutations in the IGHV1 usually have a better prognosis and a longer survival than those with an unmutated profile. In addition, the same authors state that these clinical-biological associations are present in all ethnic groups, although the biased ethnic and geographic variants of the clinical course and the IGHV1 usage can vary across the population comparing the Caucasian with the Chinese population. ${ }^{3}$ We wish to point out, however, that a recently published paper by our group (Amato et al. $)^{4}$ disclosed ethnic and geographic variations in the mutational status of $I G H V 1$ among different ethnic groups. In more detail, we observed in a double cohort study of CLL patients collected at two reference centres: one in Europe (Siena, Italy) and one in Africa (Dakar, Senegal) a strong predominance of the unmutated CLL (U-CLL) in the Senegalese cohort and a striking difference in the frequencies of the usage of IGHV1. In particular, we detected that IGHV1 (IGHV1-69), IGHD3, and IGHJ6 were significantly more frequent in Senegalese patients, whereas IGHV3-30 was not only common among the Italian cohort but also limited to that cohort. Undoubtedly, our findings reflect an antigen selection process related to the ethnic, geographic and environmental background in which individuals live, providing a biological explanation for the earlier onset of the disease and the more aggressive clinical behavior of the Senegalese CLL patients compared to the Italian CLL patients. ${ }^{4,5,6,7}$ Furthermore, the interactions between the neoplastic lymphocytes responding to B- cell receptor stimulation and the microenvironment might play a key role in CLL pathogenesis. ${ }^{8,9}$ Indeed, U-CLL, the most common subtype in Africa, shows a more active BCR signalling pathway, whereas mutated CLL (M-CLL) is the most common subtype in Western countries. ${ }^{4}$ Thus, this mechanism may reflect not only intrinsic differences in clones but also different interactions with $\mathrm{T}$ cells, presumably in response to Toll-like receptor (TLR) stimulation which is characteristic of recurrent malaria infection. ${ }^{10}$ Further evidence is provided by the detection of a preferential usage of IGHV1-69 in Senegalese patients which has been shown, similar to the vast majority of unmutated immunoglobulins, to be much more broadly active against bacteria. ${ }^{11}$ This evidence points out that chronic antigenic stimulation is a pivotal driver of malignant cells in the pathogenesis of African CLL. Despite the fact that IGHV usage and the frequency of BCR subsets can vary across populations with a different incidence of CLL (Caucasian verus Chinese and Caucasian versus African), the reliability of the IGHV1 mutational status in predicting the prognosis remains undiscussed, even though IGHV1 sequencing constitutes a valid guide for making the choice between chemoimmunotherapy and novel agents in all CLL patients requiring first-line treatment, in particular for those belonging to ethnic groups at risk. However, in the era of immunotherapy, innovative therapeutic approaches might overcome the prognostic impact of the IGHV1 mutational profile on the basis of advancements in the understanding of the CLL pathology.

Teresa Amato, Massimo Granai, Lorenzo Leoncini and Cristiana Bellan

Section of Pathology, Department of Medical Biotechnology, University of Siena, Siena, Italy

Correspondence: CRISTIANA BELLAN

bellan@unisi.it

doi:10.3324/haematol.2019.219618

Information on authorship, contributions, and financial \& other disclosures was provided by the authors and is available with the online version of this article at WwW. haematologica.org.

\section{References}

1. Giudice ID, Foà R. Another step forward in the 20-year history of IGHV mutations in chronic lymphocytic leukemia. Haematologica. 2019;104(2):219-221.

2. Baliakas P, Moysiadis T, Hadzidimitriou A, et al. Tailored approaches grounded on immunogenetic features for refined prognostication in chronic lymphocytic leukemia. Haematologica. 2019;104(2):360-369.

3. Marinelli M, Ilari C, Xia Y, et al. Immunoglobulin gene rearrangements in Chinese and Italian patients with chronic lymphocytic leukemia. Oncotarget. 2016;7(15):20520-20531.

4. Amato T, Sall A, Dièye TN, et al. Preferential Usage of Specific Immunoglobulin Heavy Chain Variable Region Genes With Unmutated Profile and Advanced Stage at Presentation Are Common Features in Patients With Chronic Lymphocytic Leukemia From Senegal. Am J Clin Pathol. 2017;148(6):545-554.

5. Mukiibi JM, Paul B, Nyirenda CM, et al. Chronic lymphocytic leukaemia (CLL) in central Africans. Cent Afr J Med. 2004;50:111115.

6. Salawu L, Bolarinwa RA, Durosinmi MA. Chronic lymphocytic leukaemia: a-twenty-years experience and problems in Ile-Ife, SouthWestern Nigeria. Afr Health Sci. 2010;10:187-192.

7. Sall A, Touré AO, Sall FB, et al. Characteristics of chronic lymphocytic leukemia in Senegal. BMC Hematol. 2016;16:10.

8. Kostareli E, Gounari M, Agathangelidis A, et al. Immunoglobulin gene repertoire in chronic lymphocytic leukemia: insight into antigen selection and microenvironmental interactions. Mediterr J Hematol Infect Dis. 2012;4:e2012052.

9. Kipps TJ, Stevenson FK, Wu CJ, et al. Chronic lymphocytic leukaemia. Nat Rev Dis Primers. 2017;3:16096.

10. Tarnani M, Laurenti L, Longo PG, et al. The proliferative response to cpg-ODN stimulation predicts PFS, TTT and OS in patients with chronic lymphocytic leukemia. Leuk Res. 2010;34(9):1189-1194.

11. Hatzi K, Catera R, Atanasio CM, et al. Chronic lymphocytic leukemia immunoglobulins display bacterial reactivity that converges and diverges from auto-/poly-reactivity and IGHV mutation status. Clin Immunol. 2016;172:44-51. 